\title{
Vesical Imaging-Reporting and Data System (VI-RADS) incorporated into bladder cancer clinical practice: what's the perspectives beyond diagnostic accuracy?
}

\author{
Francesco Del Giudice ${ }^{1,2}$, Riccardo Campa ${ }^{3}$, Marco Bicchetti $^{3}$, Ettore De Berardinis ${ }^{1}$, Valeria Panebianco ${ }^{3}$ \\ ${ }^{1}$ Department of Maternal-Infant and Urological Sciences, "Sapienza” Rome University, Policlinico Umberto I Hospital, Rome, Italy; ${ }^{2}$ Department \\ of Urology, Stanford University, School of Medicine, Stanford, CA, USA; ${ }^{3}$ Department of Radiological, Oncological and Anatomopathological \\ Sciences, "Sapienza” Rome University, Policlinico Umberto I Hospital, Rome, Italy \\ Correspondence to: Valeria Panebianco, MD. Department of Radiological, Oncological and Anatomopathological Sciences, "Sapienza” Rome \\ University, Policlinico Umberto I Hospital, Viale del Policlinico 155, 00161, Rome, Italy. Email: valeria.panebianco@uniroma1.it. \\ Response to: Wong BS, Duran C, Williams SB. Vesical imaging reporting and data system (VI-RADS) and impact on identifying depth of invasion \\ with subsequent management in bladder cancer patients: ready for prime time? Transl Androl Urol 2020. doi: 10.21037/tau-20-839.
}

Submitted May 16, 2020. Accepted for publication Jun 19, 2020.

doi: $10.21037 /$ tau-2020-07

View this article at: http://dx.doi.org/10.21037/tau-2020-07

In this issue of Translational Andrology and Urology, Wong and colleagues (1) summarize the available data regarding the newly introduced Vesical Imaging-Reporting and Data System (VI-RADS). The aim of this scoring system is to standardize bladder multiparametric magnetic resonance imaging (mpMRI) for clinical and research purposes. In particular, the original concept was developed to create a systematic approach to reporting bladder MRI and defining the risk of muscle invasion in bladder cancer [e.g., nonmuscle invasive (NMIBC) versus muscle invasive (MIBC)] (2).

From its first clinical validation for diagnostic performance in MIBC detection by Barchetti et al. (3), further reports have been published in a relatively short range of time suggesting active clinical interest in this MRI-based scoring system (4,5). Moreover, two recent meta-analysis have reported a cumulative area under the receiver operating characteristic curve (AUC) of 0.94 (95\% CI, 0.91-0.95) and 0.93 (95\% CI, 0.91-0.95) suggesting high reliability for the primary outcome of differentiating NMIBC from MIBC (6,7). While all these data are promising, they should be considered preliminary in absence of a multicenter prospective trial in order to consolidate the role of VI-RADS prior to transurethral resection of bladder tumor (TURBT).

Nevertheless, looking back at the historical course of a similar scoring system, PI-RADS (Prostate ImagingReporting and Data system) for prostate cancer (PCa) (8), there may be more potential applications than pre-operative differentiation in clinical stage. Indeed, MRI is now being used for serial monitoring of disease progression in patients during active surveillance (AS) for low-risk PCa, to guide focal therapy, and to plan surgery in high-risk disease.

When looking at the possible clinical applications of VIRADS, our group explored the utility of it for identifying understaged patients among patients that are candidates for secondary resection (Re-TURBT) (9). A recent metaanalysis has questioned the clinical usefulness of ReTURBT, reporting an overall insignificant impact of procedure on each single survival outcome (RFS, PFS, CSS, and OS) with regard of T1 NMIBC (10). VI-RADS in this setting may therefore help in accurate pre-TURBT identification of those patients who could avoid secondary resection as they are low risk for being understaged (e.g., VI-RADS score 1-2, unifocal, small tumors) versus those that are high risk (e.g., VI-RADS 4-5, diagnosed with TaT1 at initial TURBT).

Additionally, although still preliminary, VI-RADS is under evaluation as an alternative to replace deep TURBT resection to stage BCa. The "Bladder Path" trial (https:// www.birmingham.ac.uk/research/crctu/trials/Bladder-Path/ index.aspx) is testing the hypothesis as to whether TURBT can be substituted by mpMRI for addressing patients directly to radical interventions. If the preliminary results of such experience are promising, this could represent a paradigm shift 
in the algorithm of bladder cancer treatment incorporating mpMRI as a key feature the initial hematuria work-up.

Already proven in a post-hoc analysis from the PURE-01 trial (11), the role of mpMRI for assessment of pathological complete response (CR; pT0) after neoadjuvant pembrolizumab demonstrated to be reliable in predicting this critical prognostic outcome, thus offering a radiationfree alternative to the current CT-based guidelines to asses patient's response to treatment. However, this experience was limited to the sole use of neoadjuvant systemic immunotherapy and each mpMRI sequence consisted of a dichotomous variable (yes $v s$. no) which is limiting as treatment response is a spectrum. VI-RADS could represent the consequent evolution of such approach, potentially reflecting a on a 5-point scale, different degree of radiologic response therefore becoming an imaging biomarker in the context of NAC. This may finally allow for personalizing of a patient's therapeutic options rather than proceeding to cystectomy after NAC as is currently standard.

A last proof of VI-RADS versatility is from the recent proposal to adapt these criteria in the context of the COVID-19 pandemic as a risk-adapted decision-making tool for minimizing patient's risk exposure to viral infection and thus providing a prognostic criterion for adjusting oncologic class priority among overwhelmed waiting lists (12).

In conclusion, VI-RADS has potential for staging bladder cancer, but further prospective, large multi-institutional collaborations are mandatory before wide-spread adoption for local staging differentiation and also, ideally, within nomograms for predicting patient's outcome.

\section{Acknowledgments}

Funding: None.

\section{Footnote}

Provenance and Peer review: This article was commissioned by the editorial office, Translational Andrology and Urology. The article did not undergo external peer review.

Conflicts of Interest: All authors have completed the ICMJE uniform disclosure form (available at http://dx.doi. org/10.21037/tau-2020-07). The authors have no conflicts of interest to declare.

Ethical Statement: The authors are accountable for all aspects of the work in ensuring that questions related to the accuracy or integrity of any part of the work are appropriately investigated and resolved.

Open Access Statement: This is an Open Access article distributed in accordance with the Creative Commons Attribution-NonCommercial-NoDerivs 4.0 International License (CC BY-NC-ND 4.0), which permits the noncommercial replication and distribution of the article with the strict proviso that no changes or edits are made and the original work is properly cited (including links to both the formal publication through the relevant DOI and the license). See: https://creativecommons.org/licenses/by-nc-nd/4.0/.

\section{References}

1. Wong BS, Duran C, Williams SB. Vesical imaging reporting and data system (VI-RADS) and impact on identifying depth of invasion with subsequent management in bladder cancer patients: ready for prime time? Transl Androl Urol 2020. doi: 10.21037/tau-20-839.

2. Panebianco V, Narumi $Y$, Altun E, et al. Multiparametric Magnetic Resonance Imaging for Bladder Cancer: Development of VI-RADS (Vesical Imaging-Reporting And Data System). Eur Urol 2018;74:294-306.

3. Barchetti G, Simone G, Ceravolo I, et al. Multiparametric MRI of the bladder: inter-observer agreement and accuracy with the Vesical Imaging-Reporting and Data System (VI-RADS) at a single reference center. Eur Radiol 2019;29:5498-506.

4. Hechler V, Rink M, Beyersdorff D, et al. The role of the vesical imaging-reporting and data system (VI-RADS) for bladder cancer diagnostics-status quo. Urologe A 2019;58:1443-50.

5. Caglic I, Panebianco V, Vargas HA, et al. MRI of Bladder Cancer: Local and Nodal Staging. J Magn Reson Imaging 2020;52:649-67.

6. Woo S, Panebianco V, Narumi Y, et al. Diagnostic Performance of Vesical Imaging Reporting and Data System for the Prediction of Muscle-invasive Bladder Cancer: A Systematic Review and Meta-analysis. Eur Urol Oncol 2020;3:306-15.

7. Luo C, Huang B, Wu Y, et al. Use of Vesical ImagingReporting and Data System (VI-RADS) for detecting the muscle invasion of bladder cancer: a diagnostic metaanalysis. Eur Radiol 2020;30:4606-14.

8. Turkbey B, Rosenkrantz AB, Haider MA, et al. Prostate Imaging Reporting and Data System Version 2.1: 2019 Update of Prostate Imaging Reporting and Data System 
Version 2. Eur Urol 2019;76:340-51.

9. Del Giudice F, Barchetti G, De Berardinis E, et al.

Prospective Assessment of Vesical Imaging Reporting and Data System (VI-RADS) and Its Clinical Impact on the Management of High-risk Non-muscle-invasive Bladder Cancer Patients Candidate for Repeated Transurethral Resection. Eur Urol 2020;77:101-9.

10. Krajewski $W$, Nowak $€$, Poletajew $S$, et al. The Impact of Restaging Transurethral Resection of Bladder Tumor on Survival Parameters in T1 Nonmuscle-Invasive Bladder Cancer: Systematic Review and Meta-Analysis. J Endourol

Cite this article as: Del Giudice F, Campa R, Bicchetti M, De Berardinis E, Panebianco V. Vesical Imaging-Reporting and Data System (VI-RADS) incorporated into bladder cancer clinical practice: what's the perspectives beyond diagnostic accuracy? Transl Androl Urol 2020;9(5):2320-2322. doi:10.21037/ tau-2020-07
2020;34:795-804.

11. Necchi A, Bandini M, Calareso G, et al. Multiparametric Magnetic Resonance Imaging as a Noninvasive Assessment of Tumor Response to Neoadjuvant Pembrolizumab in Muscle-invasive Bladder Cancer: Preliminary Findings from the PURE-01 Study. Eur Urol 2020;77:636-43.

12. Panebianco V, Del Giudice F, Leonardo C, et al. VIRADS Scoring Criteria for Alternative Risk-adapted Strategies in the Management of Bladder Cancer During the COVID-19 Pandemic. Eur Urol 2020;78:e18-20. 